\title{
Reply to the "Remarks by Donald H. Menzel With Reference to Bailey's Comments on Solar Electric Fields ${ }^{\prime \prime}$
}

\author{
V. A. Bailey \\ Department of Physics, University of Sydney, Sydney, Australia
}

\section{Introduction}

Since Professor Menzel's "Remarks" were published without my prior knowledge, it was impossible for me to reply to them in the proper place, namely, in the same NBS Technical Note. I am therefore grateful to the Editor of "Radio Science" for permitting" me this opportunity to discuss Menzel's "Remarks."

As I do not expect that these "Remarks" will be reproduced here in toto, I shall first endeavor to summarize them clearly. This task is made somewhat difficult by reason of Menzel's form of presentation.

\section{Summary of Menzel's "Remarks"}

These are set out in six paragraphs, nearly as follows:

Paragraph 1. "Bailey has postulated the existence of a large negative electric charge on the sun and refers to apparent experimental verification of his hypothesis."

Paragraphs 2 and 3. "I completely disagree with Professor Bailey." Menzel then uses orthodox arguments in an attempt to prove that the sun's surface potential cannot exceed $2000 \mathrm{~V}$ if positive or $1.08 \mathrm{~V}$ if negative.

Paragraph 4. "These potentials are many orders of magnitude smaller than those postulated by Bailey, $10^{17} \mathrm{~V}$ or higher. No process . . . could possibly reconcile this disagreement."

Paragraph 5. "Bailey has based his conclusion on the postulate that cosmic rays energies occasionally attain the figure of $10^{17}$ electron volts. But if cosmic rays actually derived these energies by falling through a solar electric field, they would be highly directional. One concludes that the most energetic cosmic rays do not derive from solar phenomena."

Paragraph 6. Here Menzel apparently tries to argue that the magnetic fields measured by "the space probes that Bailey refers to" cannot be "the result of a rotating charged sun, "but must be due only to "high electric currents" in the sun which are "galvanic in character."

1 Published in NBS Technical Note No. 211, 3, 61, Apr. 19, 1964.

\section{Comments on Menzel's "Remarks"}

The "experimental verification," referred to in Menzel's paragraph 1, strictly applies only to the three predictions which arose from the unorthodox hypothesis that the sun carries a large negative electric charge. Also, there has not yet been published any quantitative orthodox theory which accounts for the same predicted phenomena. Hence the unorthodox hypothesis must hold the field until a better one can be found.

In his paragraphs 2, 3, and 4, Menzel bases his arguments solely on orthodox ideas. This is equivalent to saying that the unorthodox hypothesis is wrong because it is not orthodox. Menzel forgets that nearly all of the fundamental theories in physics and astronomy when first propounded have clashed with the current orthodox theories. Examples are Newton's Theory of Gravitation, Huyghen's Theory of Light, Maxwell's Theory of the Electromagnetic Field, Planck's Quantum Theory, and Einstein's Theory of Relativity.

In his paragraph 5, Menzel's assertion, "if cosmic rays actually derived these energies by falling through a solar electric field, they would be highly directional," is in general quite wrong, for the most energetic cosmic ray nuclei come from very distant regions where they possess velocities with random components transverse to the direction of the sun. These components arise from encounters with distant fields or matter, including other charged stars. Hence, like comets, these nuclei would only rarely fall radially towards the sun (as Menzel asserts) and, in general, would be distributed isotropically relative to the earth. ${ }^{2}$

The view expressed in his paragraph 6 , that the magnetic fields measured by the space probes are entirely due to electric currents in the sun, is unconvincing since it is not supported by a single quantitative example. On the contrary, we can show that if a solar current $i$ in the equatorial plane is chosen such that its equivalent magnetic moment vector $M$ generates, near the earth's orbit, the

2 Of course the theory of their orbits would have to be relativistic, so these orbits would be more complicated than ellipses, parabolas, or hyperbolas. 
quiet time magnetic field vector $B_{i}$ observed by Pioneer 5 [Bailey, 1963] then it would simultaneously generate near the sun's north pole a field vector $H_{p}$ which (a) is about 300 or more times as large as the north polar field observed recently by H. D. Babcock and (b) opposes the direction of that observed field. These facts would seem to demonstrate that Menzel's view is untenable.

\section{Conclusion}

To avoid the time-wasting business of opposing orthodox and faulty arguments with more logical arguments based on the magnetic observations made by means of the four satellites, I refer the reader to a recently published, crucial, experiment method [Bailey, 1964] of determining the truth or falsehood of the unorthodox hypothesis.
This method involves the use of two neighboring but differently moving satellites, each carrying a magnetometer. The theory of the method is entirely orthodox, and consequently the conclusions derived by means of it should command universal assent.

\section{References}

Bailey, V. A. (Sept. 14, 1963), The interplanetary magnetic field, Nature 199, 1029-1031.

Bailey, V.A. (Mar. 21, 1964), The sun's electrical charge, Nature 201, 1202-1203. 